\title{
SOFT ALMOST $\beta$-CONTINUITY IN SOFT TOPOLOGICAL SPACES
}

Alpa Singh Rajput ${ }^{1 *}$, S. S. Thakur ${ }^{2}$, Om Prakash Dubey ${ }^{3}$

${ }^{1 *}$ Research Scholar, Jabalpur Engineering College, Jabalpur, Madhya Pradesh, India; ${ }^{2}$ Principal, Jabalpur Engineering College, Jabalpur, Madhya Pradesh, India; ${ }^{3}$ Department of Mathematics, J. J. College, Veer Kunwar Singh University, Ara, Bihar, India.

Email: ${ }^{*}$ alpasinghrajput09@gmail.com, 22samajh_singh@rediffmail.com, 30mprakashdubeymaths@gmail.com Article History: Received on $12^{\text {th }}$ April 2020, Revised on $25^{\text {th }}$ May 2020, Published on $16^{\text {th }}$ June 2020

\section{Abstract}

Purpose: In the present paper the concept of soft almost $\beta$-continuous mappings and soft almost $\beta$-open mappings in soft topological spaces have been introduced and studied.

Methodology: This notion is weaker than both soft almost pre-continuous mappings, soft almost semi-continuous mapping. The diagrams of implication among these soft classes of soft mappings have been established.

Main Findings: We extend the concept of almost $\beta$-continuous mappings and almost $\beta$-open mappings in soft topology.

Implications: Mapping is an important and major area of topology and it can give many relationships between other scientific areas and mathematical models. This notion captures the idea of hanging-togetherness of image elements in an object by assigning strength of connectedness to every possible path between every possible pair of image elements. It is an important tool for the designing of algorithms for image segmentation.

The novelty of Study: Hope that the concepts and results established in this paper will help the researcher to enhance and promote the further study on soft topology to carry out a general framework for the development of information systems.

Keywords: Soft Regular Open Set, Soft $\beta$-open Set, Soft Almost Continuous Mappings, Soft $\beta$-continuous Mappings, Soft Almost $\beta$-continuous Mappings, Soft Almost $\beta$-open Mappings.

\section{INTRODUCTION}

The soft set theory was proposed by Molodtsov, D. (1999). It offers a rigid mathematical theory to deal with uncertainties. Unlike the probability theory and the fuzzy set theory suffering from the inadequacy of the parameterization tools, the soft set theory is free of those problems. In (Shabir, M. \& Naz, M. (2011)) initiated the study of soft topological spaces. Many researchers worked on the findings of structures of soft set theory, soft topology, and applied to many problems having uncertainties. Theoretical studies of soft sets and soft topological spaces have been studied in ((Ali, I. M. et al., (2009)), (Hussain, S. \& Ahmad, B. (2011)), (Kharral, A. \& Ahmad, B. (2011)), (Maji, P. K. et al., (2003)), (Majumdar, P. \& Samanta, S. K. (2008)), (Min, W. K. (2011)), (Molodtsov, D. (1999)), (Shabir, M. \& Naz, M. (2011)), (Saziye, Y. (2014)), (Zorlutuna, I. \& Hatice, C. (2015))). The concepts soft regular-open (Arockiarani, I. \& Lancy, A. A. (2013)), soft semi-open (Mahanta, J. \& Das, P. K. (2014)), soft preopen (Akdag, M. \& Ozkan A. (2014A)), soft $\alpha$-open (Akdag, M. \& Ozkan A. (2014B)) and soft $\beta$-open set (Akdag, M. \& Ozkan, A. (2014C)) play an important role in generalizations of continuity in soft topological spaces. In the present paper, we introduce the concept of soft almost $\beta$-continuous and soft almost $\beta$-open mappings and studied some of their properties and characterizations.

\section{Preliminaries}

Let $\mathrm{U}$ is an initial universe set, $\mathrm{E}$ be a set of parameters, $\mathrm{P}(\mathrm{U})$ denote the power set of $\mathrm{U}$ and $\mathrm{A} \subseteq \mathrm{E}$. $\mathrm{A}$ pair $(\mathrm{F}, \mathrm{A})$ is called a soft set over $\mathrm{U}$, where $\mathrm{F}$ is a mapping given by $\mathrm{F}: \mathrm{A} \rightarrow \mathrm{P}(\mathrm{U})$. In other words, a soft set over $\mathrm{U}$ is a parameterized family of subsets of the universe $\mathrm{U}$. For all $\mathrm{e} \in \mathrm{A}, \mathrm{F}(\mathrm{e})$ may be considered as the set of e-approximate elements of the soft set (F, A). Throughout this paper $\mathrm{S}(\mathrm{X}, \mathrm{E}), \mathrm{S}(\mathrm{Y}, \mathrm{K})$ means the family of all soft sets over X and Y respectively. For the soft set theory and soft topology the readers should refers ((Ali, I. M. et al., (2009)), (Kharral, A. \& Ahmad, B. (2011)), (Maji, P. K. et al., (2003)), (Majumdar, P. \& Samanta, S. K. (2008)), (Molodtsov, D. (1999)), ( Naz, M. (2011)), (Zorlutuna, I. \& Hatice, C. (2015)))

Definition 1: ((Akdag, M. \& Ozkan, A. (2014A)), (Akdag, M. \& Ozkan, A. (2014C)), (Arockiarani, I. \& Lancy, A. A. (2013)), (Chen, B. (2013)), (Saziye, Y. (2014))) A soft set (F, E) in a soft topological space (X, $\tau$, E) is said to be Soft regular open(resp. soft $\alpha$-open, soft semi-open, soft pre-open, soft $\beta$-open) if $(\mathrm{F}, \mathrm{E})=\operatorname{Int}(\mathrm{Cl}(\mathrm{F}, \mathrm{E}))(\mathrm{resp}$. $(\mathrm{F}, \mathrm{E}) \subseteq$ $\operatorname{Int}(\mathrm{Cl}(\operatorname{Int}(\mathrm{F}, \mathrm{E}))),(\mathrm{F}, \mathrm{E}) \subseteq \mathrm{Cl}(\operatorname{Int}(\mathrm{F}, \mathrm{E})),(\mathrm{F}, \mathrm{E}) \subseteq \operatorname{Int}(\mathrm{Cl}(\mathrm{F}, \mathrm{E})),(\mathrm{A}, \mathrm{E}) \subset \mathrm{Cl}(\operatorname{Int}(\mathrm{Cl}(\mathrm{A}, \mathrm{E}))))$.

The complement of a soft $\alpha$-open set (resp. soft semi-open set, soft pre-open, soft $\beta$-open) is called Soft $\alpha$-closed set(resp. soft semi-closed, soft pre-closed, soft $\beta$-closed).

Remark: ((Akdag M. \& Ozkan A. (2014A)), (Akdag, M. \& Ozkan, A. (2014B)), (Saziye, Y. (2014))) (a) Every soft regular open (resp. soft regular closed) set is soft open (resp. closed), every soft open (resp. soft closed) is soft $\alpha$-open (resp. soft $\alpha$-closed), every soft $\alpha$-open (resp. soft $\alpha$-closed) set is soft pre-open (resp. pre-closed) and soft semi-open (resp. semi-closed) but the converses may not be true. (b)The concept of soft semi-open (resp. soft semi-closed) and soft 
pre-open (resp. soft pre-closed) sets are independent of each other. (c) Every soft pre-open (resp. pre-closed) and soft semi-open (resp. semi-closed) set is soft $\beta$-open ((resp. soft $\beta$-closed) but the converses may not be true.

Definition 2: (Akdag, M. \& Ozkan, A. (2014C)) Let (F, E) be a soft set in a soft topological space (X, $\tau$, E).

(a) The soft $\beta$-closure of $(F, E)$ is defined as the smallest soft $\beta$-closed set which contains $(F, E)$ and it is denoted by $\beta \mathrm{Cl}(\mathrm{F}, \mathrm{E})$.

(b) The soft $\beta$-interior of (F, E) is defined as the largest soft $\beta$-open set which is contained in $(F, E)$ and is denoted by $\beta \operatorname{Int}(\mathrm{F}, \mathrm{E})$.

Definition 3: (Zorlutana, I. et al., (2012)) A soft mapping fpu : $(\mathrm{X}, \tau, \mathrm{E}) \rightarrow(\mathrm{Y}, v, \mathrm{~K})$ is said to be soft $\beta$-irresolute if $f_{p u}^{-1}$ $(\mathrm{G}, \mathrm{K})$ is soft $\beta$-open over $\mathrm{X}$, for all soft $\beta$-open set $(\mathrm{G}, \mathrm{K})$ over $\mathrm{Y}$.

Definition 4: ((Akdag, M. \& Ozkan, A. (2014B)), (Akdag, M. \& Ozkan, A. (2014C)), (Arockiarani, I. \& Lancy, A. A. (2013)), (Chen, B. (2013))) Let (X, $\tau$, E) and (Y, v, K) be a soft topological space. A soft mapping fpu : $(\mathrm{X}, \tau, \mathrm{E}) \rightarrow(\mathrm{Y}$, $v, \mathrm{~K}$ ) is said to be soft open(resp. soft $\alpha$-open, soft semi-open, soft pre-open, soft $\beta$-open) mapping if fpu (F, E) is soft open(resp. soft $\alpha$-open, soft semi-open, soft pre-open, soft $\beta$-open) over $Y$, for all soft open set (F, E) over X.

Remark: ((Akdag, M. \& Ozkan, A. (2014B)), (Akdag, M. \& Ozkan, A. (2014C))) (a) Every soft continuous (resp. soft open) mapping is soft $\alpha$-continuous (resp. soft $\alpha$-open) mapping, every soft $\alpha$-continuous (resp. soft $\alpha$-open) mapping is soft pre-continuous (resp. soft pre-open) and soft semi-continuous (resp. soft semi-open) mapping but the converse may not be true. (b) The concept of soft semi-continuous and soft pre-continuous (resp. soft semi-open and soft pre-open) mappings are independent. (c)Every soft pre-continuous (resp. soft pre-open) and soft semi-continuous (resp. soft semiopen) mapping is soft $\beta$-continuous (resp. soft $\beta$-open) mapping but the converse may not be true.

Definition 4: ((Thakur, S.S. \& Rajput, A.S. (2017A)), (Thakur S.S. \& Rajput, A.S. (2017B)), (Thakur S.S. \& Rajput, A.S. (2018)) A soft mapping fpu $:(\mathrm{X}, \tau, \mathrm{E}) \rightarrow(\mathrm{Y}, \vartheta, \mathrm{K})$ is said to be soft almost continuous (resp. $\alpha$-continuous, semicontinuous, pre-continuous) mapping if the inverse image of every soft regular open set over $\mathrm{Y}$ is soft open(soft $\alpha$-open, soft semi-open, soft pre-open) over X.

Definition 5: ((Thakur, S.S. \& Rajput, A.S. (2017A)), (Thakur, S.S. \& Rajput, A.S. (2019)), (Thakur S.S. \& Rajput, A.S. (2017B)), (Thakur S.S. \& Rajput, A.S. (2018))) A soft mapping fpu : $(\mathrm{X}, \tau, \mathrm{E}) \rightarrow(\mathrm{Y}, \vartheta, \mathrm{K})$ is said to be soft almost open (resp. $\alpha$-open, semi-open, pre-open) mapping if the image of every soft regular open set over $\mathrm{X}$ is soft open (soft $\alpha$ open, soft semi-open, soft pre-open) over Y.

Remark: ((Thakur, S.S. \& Rajput, A.S. (2017A)), (Thakur S.S. \& Rajput, A.S. (2017B)), (Thakur S.S. \& Rajput, A.S. (2018)) Every soft continuous (open) mapping is soft almost continuous (open), every soft almost continuous (resp. soft almost-open) mapping is soft almost $\alpha$-continuous (resp. soft almost $\alpha$-open), every soft $\alpha$-continuous (resp. $\alpha$-open) mapping is soft almost $\alpha$-continuous (resp. $\alpha$-open), every soft almost $\alpha$-continuous (resp. soft almost $\alpha$-open) mapping is almost pre-continuous (resp. soft almost pre-open), every soft semi-continuous mapping (resp. soft semi-open) is soft almost semi-continuous (resp. soft almost semi-open) and every soft pre-continuous (resp. soft pre-open) mapping is soft almost pre-continuous (resp. soft almost pre-open) but the converse may not be true.

Definition 6: (Thakur S.S. \& Rajput, A.S. (2018)) A soft topological space (X, $\tau$, E) is said to be soft semi-regular if for each soft open set $(F, E)$ and each soft point $(x e)_{E} \in(F, E)$, there exists a soft open set $(G, E)$ such that $(x e)_{E} \in(G, E)$ and $(\mathrm{G}, \mathrm{E}) \subset \operatorname{Int}(\mathrm{Cl}(\mathrm{G}, \mathrm{E})) \subset(\mathrm{F}, \mathrm{E})$.

\section{Soft Almost $\beta$-Continuous Mappings}

Definition 1: A soft mapping fpu: $(X, \tau, E) \rightarrow(Y, \vartheta, K)$ is said to be soft almost $\beta$-continuous if the inverse image of every soft regular open set over $Y$ is soft $\beta$-open over $X$.

Remark: Every soft $\beta$-continuous mapping is soft almost $\beta$-continuous but the converse may not be true.

Example 1: Let $X=\{x 1, x 2\}, E=\{e 1, e 2\}$ and $Y=\{y 1, y 2\}, K=\{k 1, k 2\}$. The soft sets $(F, E),(G, K)$ are defined as follows : $\mathrm{F}(\mathrm{e} 1)=\{\mathrm{x} 2\}, \mathrm{F}(\mathrm{e} 2)=\{\mathrm{x} 1\}, \mathrm{G}(\mathrm{k} 1)=\{\mathrm{y} 1\}, \mathrm{G}(\mathrm{k} 2)=\{\mathrm{y} 2\}$. Let $\tau=\{\varphi,(\mathrm{F}, \mathrm{E}), \mathrm{X}\}$ and $v=\{\varphi,(\mathrm{G}, \mathrm{K}), \mathrm{Y}\}$ are topologies on $\mathrm{X}$ and $\mathrm{Y}$ respectively. Then soft mapping fpu: $(X, \tau, E) \rightarrow(Y, v, K)$ defined by $u(x 1)=y 1, u(x 2)=y 2$ and $\mathrm{p}(\mathrm{e} 1)=\mathrm{k} 1, \mathrm{p}(\mathrm{e} 2)=\mathrm{k} 2$ is soft almost $\beta$-continuous mapping but not soft $\beta$-continuous.

Remark: Every soft almost semi-continuous mapping is soft almost $\beta$-continuous but the converse may not be true.

Example 2: Let $\mathrm{X}=\{\mathrm{x} 1, \mathrm{x} 2\}, \mathrm{E}=\{\mathrm{e} 1, \mathrm{e} 2\}$ and $\mathrm{Y}=\{\mathrm{y} 1, \mathrm{y} 2\}, \mathrm{K}=\{\mathrm{k} 1, \mathrm{k} 2\}$. The soft sets $(\mathrm{G} 1, \mathrm{~K})$ and $(\mathrm{G} 2, \mathrm{~K})$ are defined as follows: $\mathrm{G} 1(\mathrm{k} 1)=\{\mathrm{y} 1\}, \mathrm{G} 1(\mathrm{k} 2)=\{\mathrm{y} 2\}, \mathrm{G} 2(\mathrm{k} 1)=\{\mathrm{y} 2\}, \mathrm{G} 2(\mathrm{k} 2)=\{\mathrm{y} 1\}$. Let $\tau=\{\varphi, \mathrm{X}\}$ and $v=\{\varphi,(\mathrm{G} 1, \mathrm{~K})$, $(\mathrm{G} 2, \mathrm{~K}), \mathrm{Y}\}$ are topologies on $\mathrm{X}$ and $\mathrm{Y}$ respectively. Then soft mapping fpu: $(\mathrm{X}, \tau, \mathrm{E}) \rightarrow(\mathrm{Y}, \mathrm{v}, \mathrm{K})$ defined by $\mathrm{u}(\mathrm{x} 1)=\mathrm{y} 1$ , $\mathrm{u}(\mathrm{x} 2)=\mathrm{y} 2$ and $\mathrm{p}(\mathrm{e} 1)=\mathrm{k} 1, \mathrm{p}(\mathrm{e} 2)=\mathrm{k} 2$ is soft almost $\beta$-continuous mapping but not soft almost semi-continuous.

Remark: Every soft almost pre-continuous mapping is soft almost $\beta$-continuous but the converse may not be true. 
Example 3: Let $X=\{x 1, x 2\}, E=\{e 1, e 2\}$ and $Y=\{y 1, y 2\}, K=\{k 1, k 2\}$. The soft sets $(F 1, E),(F 2, E),(F 3, E),(G 1$, $\mathrm{K})$ and $(\mathrm{G} 2, \mathrm{~K})$ are defined as follows: $\mathrm{F} 1(\mathrm{e} 1)=\varphi, \mathrm{F} 1(\mathrm{e} 2)=\{\mathrm{x} 1\}, \mathrm{F} 2(\mathrm{e} 1)=\{\mathrm{x} 1\}, \mathrm{F} 2(\mathrm{e} 2)=\varphi, \mathrm{F} 3(\mathrm{e} 1)=\{\mathrm{x} 1\}, \mathrm{F} 3(\mathrm{e} 2)=$ $\{\mathrm{x} 1\}, \mathrm{G} 1(\mathrm{k} 1)=\{\mathrm{y} 1\}, \mathrm{G} 1(\mathrm{k} 2)=\{\mathrm{y} 2\}, \mathrm{G} 2(\mathrm{k} 1)=\{\mathrm{y} 2\}, \mathrm{G} 2(\mathrm{k} 2)=\{\mathrm{y} 1\}$. Let $\tau=\{\varphi,(\mathrm{F} 1, \mathrm{E}),(\mathrm{F} 2, \mathrm{E}),(\mathrm{F} 3, \mathrm{E}), \mathrm{X}\}$ and $v=$ $\{\varphi,(\mathrm{G} 1, \mathrm{~K}),(\mathrm{G} 2, \mathrm{~K}), \mathrm{Y}\}$ are topologies on $\mathrm{X}$ and $\mathrm{Y}$ respectively. Then soft mapping fpu: $(\mathrm{X}, \tau, \mathrm{E}) \rightarrow(\mathrm{Y}, v, \mathrm{~K}) \mathrm{defined}$ by $\mathrm{u}(\mathrm{x} 1)=\mathrm{y} 1, \mathrm{u}(\mathrm{x} 2)=\mathrm{y} 2$ and $\mathrm{p}(\mathrm{e} 1)=\mathrm{k} 1, \mathrm{p}(\mathrm{e} 2)=\mathrm{k} 2$ is soft almost $\beta$-continuous mapping but not soft almost precontinuous.

Remark: Every soft almost $\alpha$-continuous mapping is soft almost semi-continuous but the converse may not be true.

Example 4: Let $X=\{x 1, x 2\}, E=\{e 1, e 2\}$ and $Y=\{y 1, y 2\}, K=\{k 1, k 2\}$. The soft sets $(F 1, E),(F 2, E),(F 3, E),(G 1$, $\mathrm{K})$ and $(\mathrm{G} 2, \mathrm{~K})$ are defined as follows: $\mathrm{F} 1(\mathrm{e} 1)=\varphi, \mathrm{F} 1(\mathrm{e} 2)=\{\mathrm{x} 1\}, \mathrm{F} 2(\mathrm{e} 1)=\{\mathrm{x} 1\}, \mathrm{F} 2(\mathrm{e} 2)=\varphi, \mathrm{F} 3(\mathrm{e} 1)=\{\mathrm{x} 1\}, \mathrm{F} 3(\mathrm{e} 2)=$ $\{\mathrm{x} 1\}, \mathrm{G} 1(\mathrm{k} 1)=\{\mathrm{y} 1\}, \mathrm{G} 1(\mathrm{k} 2)=\{\mathrm{y} 2\}, \mathrm{G} 2(\mathrm{k} 1)=\{\mathrm{y} 2\}, \mathrm{G} 2(\mathrm{k} 2)=\{\mathrm{y} 1\}$. Let $\tau=\{\varphi,(\mathrm{F} 1, \mathrm{E}),(\mathrm{F} 2, \mathrm{E}),(\mathrm{F} 3, \mathrm{E}), \mathrm{X}\}$ and $v=$ $\{\varphi,(\mathrm{G} 1, \mathrm{~K}),(\mathrm{G} 2, \mathrm{~K}), \mathrm{Y}\}$ are topologies on $\mathrm{X}$ and $\mathrm{Y}$ respectively. Then soft mapping fpu: $(\mathrm{X}, \tau, \mathrm{E}) \rightarrow(\mathrm{Y}, v, \mathrm{~K})$ defined by $\mathrm{u}(\mathrm{x} 1)=\mathrm{y} 1, \mathrm{u}(\mathrm{x} 2)=\mathrm{y} 2$ and $\mathrm{p}(\mathrm{e} 1)=\mathrm{k} 1, \mathrm{p}(\mathrm{e} 2)=\mathrm{k} 2$ is soft almost semi-continuous mapping but not soft almost $\alpha-$ continuous.

Remark: The concepts of soft almost semi-continuous and soft almost pre-continuous mappings are independent.

Example 5: Let $X=\{x 1, x 2\}, E=\{e 1, e 2\}$ and $Y=\{y 1, y 2\}, K=\{k 1, k 2\}$. The soft sets $(\mathrm{G} 1, \mathrm{~K})$ and $(\mathrm{G} 2, \mathrm{~K})$ are defined as follows: $\mathrm{G} 1(\mathrm{k} 1)=\{\mathrm{y} 1\}, \mathrm{G} 1(\mathrm{k} 2)=\{\mathrm{y} 2\}, \mathrm{G} 2(\mathrm{k} 1)=\{\mathrm{y} 2\}, \mathrm{G} 2(\mathrm{k} 2)=\{\mathrm{y} 1\}$. Let $\tau=\{\varphi, X\}$ and $v=\{\varphi,(\mathrm{G} 1, \mathrm{~K})$, $(\mathrm{G} 2, \mathrm{~K}), \mathrm{Y}\}$ are topologies on $\mathrm{X}$ and $\mathrm{Y}$ respectively. Then soft mapping fpu: $(\mathrm{X}, \tau, \mathrm{E}) \rightarrow(\mathrm{Y}, v, \mathrm{~K})$ defined by $\mathrm{u}(\mathrm{x} 1)=$ $\mathrm{y} 1, \mathrm{u}(\mathrm{x} 2)=\mathrm{y} 2$ and $\mathrm{p}(\mathrm{e} 1)=\mathrm{k} 1, \mathrm{p}(\mathrm{e} 2)=\mathrm{k} 2$ is soft almost pre-continuous mapping but not soft almost semi-continuous.

Example 6: Let $X=\{x 1, x 2\}, E=\{e 1, e 2\}$ and $Y=\{y 1, y 2\}, K=\{k 1, k 2\}$. The soft sets $(F 1, E),(F 2, E),(F 3, E),(G 1$, $\mathrm{K})$ and $(\mathrm{G} 2, \mathrm{~K})$ are defined as follows: $\mathrm{F} 1(\mathrm{e} 1)=\varphi, \mathrm{F} 1(\mathrm{e} 2)=\{\mathrm{x} 1\}, \mathrm{F} 2(\mathrm{e} 1)=\{\mathrm{x} 1\}, \mathrm{F} 2(\mathrm{e} 2)=\varphi, \mathrm{F} 3(\mathrm{e} 1)=\{\mathrm{x} 1\}, \mathrm{F} 3(\mathrm{e} 2)=$ $\{\mathrm{x} 1\}, \mathrm{G} 1(\mathrm{k} 1)=\{\mathrm{y} 1\}, \mathrm{G} 1(\mathrm{k} 2)=\{\mathrm{y} 2\}, \mathrm{G} 2(\mathrm{k} 1)=\{\mathrm{y} 2\}, \mathrm{G} 2(\mathrm{k} 2)=\{\mathrm{y} 1\}$. Let $\tau=\{\varphi,(\mathrm{F} 1, \mathrm{E}),(\mathrm{F} 2, \mathrm{E}),(\mathrm{F} 3, \mathrm{E}), \mathrm{X}\}$ and $v=$ $\{\varphi,(\mathrm{G} 1, \mathrm{~K}),(\mathrm{G} 2, \mathrm{~K}), \mathrm{Y}\}$ are topologies on $\mathrm{X}$ and $\mathrm{Y}$ respectively. Then soft mapping fpu: $(\mathrm{X}, \tau, \mathrm{E}) \rightarrow(\mathrm{Y}, v, \mathrm{~K}) \mathrm{defined}$ by $\mathrm{u}(\mathrm{x} 1)=\mathrm{y} 1, \mathrm{u}(\mathrm{x} 2)=\mathrm{y} 2$ and $\mathrm{p}(\mathrm{e} 1)=\mathrm{k} 1, \mathrm{p}(\mathrm{e} 2)=\mathrm{k} 2$ is soft almost semi-continuous mapping but not soft almost precontinuous.

Thus we reach the following diagram of implications.

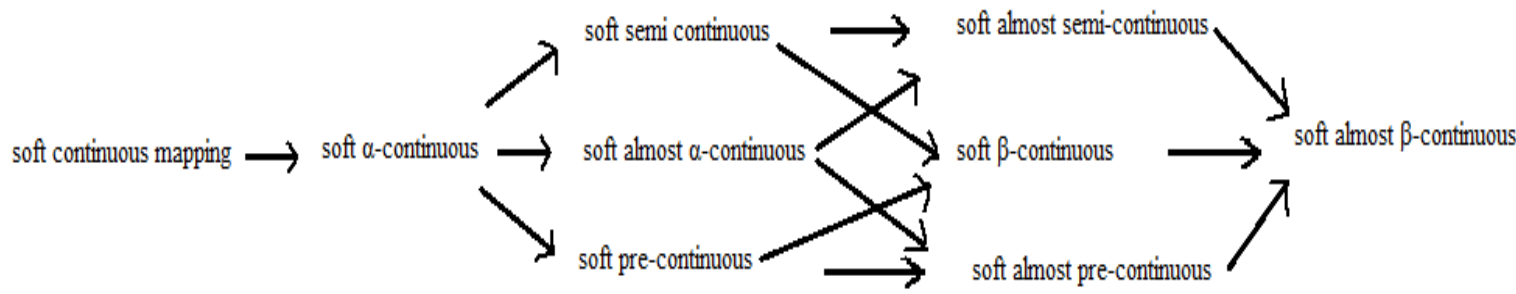

Figure 1: Soft continuous mapping

\section{Properties of Soft Almost $\beta$-Continuous Mappings}

Theorem 1: Let fpu: $(\mathrm{X}, \tau, \mathrm{E}) \rightarrow(\mathrm{Y}, \vartheta, \mathrm{K})$ be a soft mapping. Then the following conditions are equivalent:

(a) fpu is soft almost $\beta$-continuous.

(b) $f_{p u}^{-1}(\mathrm{G}, \mathrm{K})$ is soft $\beta$-closed set in $\mathrm{X}$ for every soft regular closed set $(\mathrm{G}, \mathrm{K})$ in $\mathrm{Y}$.

(c) $f_{p u}^{-1}(\mathrm{~A}, \mathrm{~K}) \subset \beta \operatorname{Int}\left(f_{p u}^{-1}(\operatorname{Int}(\mathrm{Cl}(\mathrm{A}, \mathrm{K})))\right)$ for every soft open set $(\mathrm{A}, \mathrm{K})$ in $\mathrm{Y}$.

(d) $\beta \mathrm{Cl}\left(f_{p u}^{-1}(\mathrm{Cl}(\operatorname{Int}(\mathrm{G}, \mathrm{K})))\right) \subset f_{p u}^{-1}(\mathrm{G}, \mathrm{K})$ for every soft closed set $(\mathrm{G}, \mathrm{K})$ in $\mathrm{Y}$.

(e) For each soft point (xe)E over $\mathrm{X}$ and each soft regular open set $(\mathrm{G}, \mathrm{K})$ over $\mathrm{Y}$ containing fpu ((xe)E), there exists a soft $\beta$-open set $(\mathrm{F}, \mathrm{E})$ over X such that $(\mathrm{xe}) \mathrm{E} \in(\mathrm{F}, \mathrm{E})$ and $(\mathrm{F}, \mathrm{E}) \subset f_{p u}^{-1}(\mathrm{G}, \mathrm{K})$.

(f ) For each soft point (xe)E over $\mathrm{X}$ and each soft regular open set $(\mathrm{G}, \mathrm{K})$ over $\mathrm{Y}$ containing fpu((xe)E), there exists a soft $\beta$-open set $(F, E)$ over $X$ such that $(x e) E \in(F, E)$ and fpu(F, E) $\subset(G, K)$.

Proof: $(\mathrm{a}) \Leftrightarrow$ (b) Since $f_{p u}^{-1}\left((\mathrm{G}, \mathrm{K})^{\mathrm{C}}\right)=\left(f_{p u}^{-1}(\mathrm{G}, \mathrm{K})\right)^{\mathrm{C}}$ for every soft set $(\mathrm{G}, \mathrm{K})$ over $\mathrm{Y}$.

$(\mathrm{a}) \Rightarrow(\mathrm{c})$ Since $(\mathrm{A}, \mathrm{K})$ is soft open set over $\mathrm{Y},(\mathrm{A}, \mathrm{K}) \subset \operatorname{Int}(\mathrm{Cl}(\mathrm{A}, \mathrm{K}))$ and hence $f_{p u}^{-1}(\mathrm{~A}, \mathrm{~K}) \subset f_{p u}^{-1}(\operatorname{Int}(\mathrm{Cl}(\mathrm{A}, \mathrm{K})))$. Now $\operatorname{Int}(\mathrm{Cl}(\mathrm{A}, \mathrm{K}))$ is a soft regular open set over $\mathrm{Y}$. By $(\mathrm{a}), f_{p u}^{-1}(\operatorname{Int}(\mathrm{Cl}(\mathrm{A}, \mathrm{K})))$ is soft $\beta$-open set over X. Thus $f_{p u}^{-1}(\mathrm{~A}, \mathrm{~K}) \subset$ $f_{p u}^{-1}(\operatorname{Int}(\mathrm{Cl}(\mathrm{A}, \mathrm{K})))=\beta \operatorname{Int}\left(f_{p u}^{-1}(\operatorname{Int}(\mathrm{Cl}(\mathrm{A}, \mathrm{K})))\right)$. 
(c) $\Rightarrow$ (a) Let $(\mathrm{A}, \mathrm{K})$ be a soft regular open set over $\mathrm{Y}$, then we have $f_{p u}^{-1}(\mathrm{~A}, \mathrm{~K}) \subset \beta \operatorname{Int}\left(f_{p u}^{-1}(\operatorname{Int}(\mathrm{Cl}(\mathrm{A}, \mathrm{K})))\right)=\beta \operatorname{Int}\left(f_{p u}^{-1}\right.$ $(\mathrm{A}, \mathrm{K}))$. Thus, $f_{p u}^{-1}(\mathrm{~A}, \mathrm{~K})=\beta \operatorname{Int}\left(f_{p u}^{-1}(\mathrm{~A}, \mathrm{~K})\right)$ shows that $f_{p u}^{-1}(\mathrm{~A}, \mathrm{~K})$ is a soft $\beta$-open set over $\mathrm{X}$.

(b) $\Rightarrow(\mathrm{d})$ Since $(\mathrm{G}, \mathrm{K})$ is soft closed set over $\mathrm{Y}, \mathrm{Cl}(\operatorname{Int}(\mathrm{G}, \mathrm{K})) \subset(\mathrm{G}, \mathrm{K})$ and $f_{p u}^{-1}(\mathrm{Cl}(\mathrm{Int}(\mathrm{G}, \mathrm{K}))) \subset f_{p u}^{-1}(\mathrm{G}, \mathrm{K})$. $\mathrm{Cl}(\mathrm{Int}$ $(\mathrm{G}, \mathrm{K}))$ is soft regular closed set over $\mathrm{Y}$. Hence, $f_{p u}^{-1}\left(\mathrm{Cl}\left(\mathrm{Int}(\mathrm{G}, \mathrm{K})\right.\right.$ is soft $\beta$-closed set over X. Thus, $\beta \mathrm{Cl}\left(f_{p u}^{-1}(\mathrm{Cl}\right.$ ( $\operatorname{Int}(\mathrm{G}, \mathrm{K}))))=f_{p u}^{-1}(\mathrm{Cl}(\operatorname{Int}(\mathrm{G}, \mathrm{K}))) \subset f_{p u}^{-1}(\mathrm{G}, \mathrm{K})$.

$(\mathrm{d}) \Rightarrow(\mathrm{b})$ Let $(\mathrm{G}, \mathrm{K})$ be a soft regular closed set over $\mathrm{Y}$, then we have $\beta \mathrm{Cl}\left(f_{p u}^{-1}(\mathrm{G}, \mathrm{K})\right)=\beta \mathrm{Cl}\left(f_{p u}^{-1}(\mathrm{Cl}(\operatorname{Int}(\mathrm{G}, \mathrm{K})))\right) \subset f_{p u}^{-1}$ $(\mathrm{G}, \mathrm{K})$. Thus, $\beta \mathrm{Cl}\left(f_{p u}^{-1}(\mathrm{G}, \mathrm{K})\right) \subset f_{p u}^{-1}(\mathrm{G}, \mathrm{K})$, shows that $f_{p u}^{-1}(\mathrm{G}, \mathrm{K})$ is soft $\beta$-closed set over $\mathrm{X}$.

(a) $\Rightarrow$ (e) Let (xe)E be a soft point over $X$ and $(G, K)$ be a soft regular open set over $Y$ such that fpu((xe)E) $\in(G, K)$, Put $(\mathrm{F}, \mathrm{E})=f_{p u}^{-1}(\mathrm{G}, \mathrm{K})$. Then by $(\mathrm{a}),(\mathrm{F}, \mathrm{E})$ is soft $\beta$-open set, $(\mathrm{xe}) \mathrm{E} \in(\mathrm{F}, \mathrm{E})$ and $(\mathrm{F}, \mathrm{E}) \subset f_{p u}^{-1}(\mathrm{G}, \mathrm{K})$.

(e) $\Rightarrow$ (f ) Let (xe)E be a soft point over X and (G, K) be a soft regular open set over Y such that fpu((xe)E). By (e) there exists a soft $\beta$-open set $(\mathrm{F}, \mathrm{E})$ such that $(\mathrm{xe}) \mathrm{E} \in(\mathrm{F}, \mathrm{E}),(\mathrm{F}, \mathrm{E}) \subset f_{p u}^{-1}(\mathrm{G}, \mathrm{K})$. And so, we have $(\mathrm{xe}) \mathrm{E} \in(\mathrm{F}, \mathrm{E}), \mathrm{fpu}(\mathrm{F}, \mathrm{E})$ $\subset \operatorname{fpu}\left(f_{p u}^{-1}(\mathrm{G}, \mathrm{K})\right) \subset(\mathrm{G}, \mathrm{K})$.

(f) $\Rightarrow$ (a) Let $(\mathrm{G}, \mathrm{K})$ be a soft regular open set over $\mathrm{Y}$ and (xe)E be a soft point over $\mathrm{X}$ such that $(\mathrm{xe}) \mathrm{E} \in f_{p u}^{-1}(\mathrm{G}, \mathrm{K})$. Then $\mathrm{fpu}((\mathrm{xe}) \mathrm{E}) \in \mathrm{fpu}\left(f_{p u}^{-1}(\mathrm{G}, \mathrm{K})\right) \subset(\mathrm{G}, \mathrm{K})$. By (f), there exists a soft $\beta$-open set $(\mathrm{F}, \mathrm{E})$ such that $(\mathrm{xe}) \mathrm{E} \in(\mathrm{F}, \mathrm{E})$ and fpu(F, E) $\subset(\mathrm{G}, \mathrm{K})$. This shows that $(\mathrm{xe}) \mathrm{E} \in(\mathrm{F}, \mathrm{E}) \subset f_{p u}^{-1}(\mathrm{G}, \mathrm{K})$. It follows that $f_{p u}^{-1}(\mathrm{G}, \mathrm{K})$ is soft $\beta$-open set and hence $f_{p u}^{-1}$ is soft almost $\beta$-continuous.

Definition 1: (Thakur, S.S. \& Rajput, A.S. (2019)) A soft point (xe)E in a soft topological space (X, $\tau$, E) is called $\delta$ cluster point of a soft set $(\mathrm{A}, \mathrm{E})$ of $\mathrm{X}$ if $\operatorname{Int}(\mathrm{Cl}(\mathrm{V}, \mathrm{E})) \cap(\mathrm{A}, \mathrm{E})^{\mathrm{C}}=\varphi$ for each soft open set $(\mathrm{V}, \mathrm{E})$ containing $(\mathrm{xe}) \mathrm{E}$. The union of all $\delta$-cluster points of $(\mathrm{A}, \mathrm{E})$ is called $\delta$-closure of $(\mathrm{A}, \mathrm{E})$ and is denoted by $\delta \mathrm{Cl}(\mathrm{A}, \mathrm{E})$. A soft set $(\mathrm{A}, \mathrm{E})$ is called soft $\delta$-closed if $(\mathrm{A}, \mathrm{E})=\delta \mathrm{Cl}(\mathrm{A}, \mathrm{E})$. The complement of a soft $\delta$-closed is called soft $\delta$-open.

Theorem 2: Let fpu: $(\mathrm{X}, \tau, \mathrm{E}) \rightarrow(\mathrm{Y}, \vartheta, \mathrm{K})$ be a soft mapping. Then the following conditions are equivalent:

(a) fpu is soft almost $\beta$-continuous.

(b) $\operatorname{fpu}(\beta \mathrm{Cl}(\mathrm{A}, \mathrm{E})) \subset \delta \mathrm{Cl}(\mathrm{fpu}(\mathrm{A}, \mathrm{E}))$, for every soft set $(\mathrm{A}, \mathrm{E})$ over $\mathrm{X}$.

(c) $\beta \mathrm{Cl}\left(f_{p u}^{-1}(\mathrm{~B}, \mathrm{~K})\right) \subset f_{p u}^{-1}(\delta \mathrm{Cl}(\mathrm{B}, \mathrm{K}))$, for every soft set $(\mathrm{B}, \mathrm{K})$ over $\mathrm{Y}$.

(d) $f_{p u}^{-1}(\mathrm{~F}, \mathrm{~K})$ is soft $\beta$-closed set over $\mathrm{X}$, for every soft $\delta$-closed set $(\mathrm{F}, \mathrm{K})$ over $\mathrm{Y}$.

(e) $f_{p u}^{-1}(\mathrm{~V}, \mathrm{~K})$ is soft $\beta$-open set over $\mathrm{X}$, for every soft $\delta$-open set $(\mathrm{V}, \mathrm{K})$ over $\mathrm{Y}$.

Proof: (a) $\rightarrow$ (b) Let (A, E) be a soft set over X. Since, $\delta \mathrm{Cl}(\mathrm{fpu}(\mathrm{A}, \mathrm{E}))$ is a soft $\delta$-closed set over Y. By theorem 1, we have $(\mathrm{A}, \mathrm{E}) \subset f_{p u}^{-1}(\delta \mathrm{Cl}(\mathrm{fpu}(\mathrm{A}, \mathrm{E})))$ which is soft $\beta$-closed set over $\mathrm{X}$. Hence, $\beta \mathrm{Cl}(\mathrm{A}, \mathrm{E}) \subset f_{p u}^{-1}(\delta \mathrm{Cl}(\mathrm{fpu}(\mathrm{A}, \mathrm{E})))$. Hence, we obtain fpu( $(\beta \mathrm{Cl}(\mathrm{A}, \mathrm{E})) \subset \delta \mathrm{Cl}(\mathrm{fpu}(\mathrm{A}, \mathrm{E}))$.

$(\mathrm{b}) \rightarrow(\mathrm{c})$ Let $(\mathrm{B}, \mathrm{K})$ be a soft set over $\mathrm{Y}$. We have $\mathrm{fpu}\left(\beta \mathrm{Cl}\left(f_{p u}^{-1}(\mathrm{~B}, \mathrm{~K})\right)\right) \subset \delta \mathrm{Cl}\left(\mathrm{fpu}\left(f_{p u}^{-1}(\mathrm{~B}, \mathrm{~K})\right) \subset \delta \mathrm{Cl}(\mathrm{B}, \mathrm{K})\right.$ and hence, $\beta \mathrm{Cl}\left(f_{p u}^{-1}(\mathrm{~B}, \mathrm{~K})\right) \subset f_{p u}^{-1}(\delta \mathrm{Cl}(\mathrm{B}, \mathrm{K}))$.

$(\mathrm{c}) \rightarrow(\mathrm{d})$ Let $(\mathrm{F}, \mathrm{K})$ be a soft $\delta$-closed set over $\mathrm{Y}$. We have $\beta \mathrm{Cl}\left(f_{p u}^{-1}(\mathrm{~F}, \mathrm{~K})\right) \subset f_{p u}^{-1}(\delta \mathrm{Cl}(\mathrm{F}, \mathrm{K}))=f_{p u}^{-1}(\mathrm{~F}, \mathrm{~K})$ and $f_{p u}^{-1}(\mathrm{~F}$, $\mathrm{K})$ is soft $\beta$-closed over $\mathrm{X}$.

$(\mathrm{d}) \rightarrow(\mathrm{e})$ Let $(\mathrm{V}, \mathrm{K})$ be a soft $\delta$-open set over $\mathrm{Y}$. By $(\mathrm{d})$, we have $f_{p u}^{-1}(\mathrm{~V}, \mathrm{~K})^{\mathrm{c}}=\left(f_{p u}^{-1}(\mathrm{~V}, \mathrm{~K})\right)^{\mathrm{c}}$, which is soft $\beta$-closed over $\mathrm{X}$ and so $f_{p u}^{-1}(\mathrm{~V}, \mathrm{~K})$ is soft $\beta$-open set in $\mathrm{X}$.

$(\mathrm{e}) \rightarrow\left(\right.$ a) Let $(\mathrm{V}, \mathrm{K})$ be a soft regular open set over $\mathrm{Y}$. Since $(\mathrm{V}, \mathrm{K})$ is soft $\delta$-open set over $\mathrm{Y}, f_{p u}^{-1}(\mathrm{~V}, \mathrm{~K})$ is soft $\beta$-open over $\mathrm{X}$ and hence by theorem 1 , fpu is soft almost $\beta$-continuous.

Theorem 3: Let fpu: $(X, \tau, E) \rightarrow(Y, \vartheta, K)$ be a soft mapping from a soft topological space $(X, \tau, E)$ to a soft semiregular space $(\mathrm{Y}, \vartheta, \mathrm{K})$. Then fpu is soft almost $\beta$-continuous if and only if fpu is soft $\beta$-continuous.

Proof: Necessity: Let (xe)E be a soft point over $X$ and $(F, K)$ be a soft open set over $Y$ such that fpu( (xe)E) $\in(F, K)$. Since $(Y, \vartheta, K)$ is soft semi-regular there exists a soft open set $(G, K)$ over $Y$ such that $f p u((x e) E) \in(G, K)$ and $(G, K) \subset$ $\operatorname{Int}(\mathrm{Cl}(\mathrm{G}, \mathrm{K}) \subset(\mathrm{F}, \mathrm{K})$. Since $\operatorname{Int}(\mathrm{Cl}(\mathrm{G}, \mathrm{K}))$ is soft regular open over $\mathrm{Y}$ and fpu is soft almost $\beta$-continuous, by theorem 1 (f) there exists a soft $\beta$-open set (A, E) over $X$ such that $(x e) E \in(A, E)$ and fpu(A, E) $\subset \operatorname{Int}(\mathrm{Cl}(\mathrm{G}, \mathrm{K}))$. Thus, (A, E) is soft $\beta$-open set such that $(\mathrm{xe}) \mathrm{E} \in(\mathrm{A}, \mathrm{E})$ and fpu(A, E) $\subset(\mathrm{F}, \mathrm{K})$. Hence by theorem (3) (Zorlutana, I. et al., (2012)), fpu is soft $\beta$-continuous.

Sufficiency: Obvious. 
Lemma 1: If fpu : $(\mathrm{X}, \tau, \mathrm{E}) \rightarrow(\mathrm{Y}, \vartheta, \mathrm{K})$ is a soft open and soft continuous mapping then $f_{p u}^{-1}(\mathrm{G}, \mathrm{K})$ is soft $\beta$-open over $\mathrm{X}$ for every $(\mathrm{G}, \mathrm{K})$ is soft $\beta$-open over $\mathrm{Y}$.

Proof: Let $(\mathrm{G}, \mathrm{K})$ is soft $\beta$-open over Y.Then, $(\mathrm{G}, \mathrm{K}) \subseteq \operatorname{Int}(\mathrm{Cl}(\operatorname{Int}(\mathrm{G}, \mathrm{K})))$.Since fpu is soft continuous we have, $\mathrm{f}-1 \mathrm{pu}$ $(\mathrm{G}, \mathrm{K}) \subseteq f_{p u}^{-1}(\operatorname{Int}(\mathrm{Cl}(\operatorname{Int}(\mathrm{G}, \mathrm{K})))) \subseteq \operatorname{Int}\left(f_{p u}^{-1}(\mathrm{Cl}(\operatorname{Int}(\mathrm{G}, \mathrm{K})))\right)$. By the openness of fpu, we have $f_{p u}^{-1}(\mathrm{Cl}(\operatorname{Int}(\mathrm{G}, \mathrm{K}))) \subseteq$ $\mathrm{Cl}\left(f_{p u}^{-1}(\operatorname{Int}(\mathrm{G}, \mathrm{K}))\right)$. Again fpu is soft continuous $f_{p u}^{-1}(\operatorname{Int}(\mathrm{G}, \mathrm{K})) \subseteq \operatorname{Int}\left(f_{p u}^{-1}(\mathrm{G}, \mathrm{K})\right)$. Thus, $f_{p u}^{-1}(\mathrm{G}, \mathrm{K}) \subseteq \operatorname{Int}\left(\mathrm{Cl}\left(\operatorname{Int}\left(f_{p u}^{-1}\right.\right.\right.$ $(\mathrm{G}, \mathrm{K}))))$. Consequently, $f_{p u}^{-1}(\mathrm{G}, \mathrm{K})$ is soft $\beta$-open over $\mathrm{X}$.

Theorem 4: If $f_{p 1 u 1}:(X, \tau, E) \rightarrow(Y, \vartheta, K)$ is soft open soft continuous and $g_{p 2 u 2}:(Y, \vartheta, K) \rightarrow(Z, \eta, T)$ is soft almost $\beta$ continuous, then $\mathrm{g}_{\mathrm{p} 2 \mathrm{u} 2} 0$ fp1u1: $(\mathrm{X}, \tau, \mathrm{E}) \rightarrow(\mathrm{Z}, \eta, \mathrm{T})$ is soft almost $\beta$-continuous.

Proof: Suppose $(U, T)$ is a soft regular open set over $Z$. Then $g-1_{p 2 u 2}(U, T)$ is a soft $\beta$-open set over $Y$ because $g_{p 2 u 2}$ is soft almost $\beta$-continuous. Since fp1u1 being soft open and continuous. By lemma 4.5 ( $\mathrm{f}-1$ plu1 $(\mathrm{g}-1$ p2u2 $(\mathrm{U}, \mathrm{T})$ ) is soft $\beta$-open over $\mathrm{X}$. Consequently, $\mathrm{g}_{\mathrm{p} 2 \mathrm{u} 2} 0 \mathrm{f}_{\mathrm{p} 1 \mathrm{u} 1}:(\mathrm{X}, \tau, \mathrm{E}) \rightarrow(\mathrm{Z}, \eta, \mathrm{T})$ is soft almost $\beta$-continuous.

Lemma 2: If (A, E) be a soft $\beta$-open set over $X$ and $(Y, E)$ is soft open in a soft topological space (X, $\tau, E)$. Then (A, E) $\cap(\mathrm{Y}, \mathrm{E})$ is soft $\beta$-open in $(\mathrm{Y}, \mathrm{E})$.

Proof: Obvious.

Theorem 5: Let fpu: $(\mathrm{X}, \tau, \mathrm{E}) \rightarrow(\mathrm{Y}, \vartheta, \mathrm{K})$ be a soft almost $\beta$-continuous mapping and $(\mathrm{A}, \mathrm{E})$ is soft open set over $\mathrm{X}$, Then $\mathrm{fpu} / \mathrm{A}, \mathrm{E})$ is soft almost $\beta$-continuous.

Proof: Let $(G, K)$ be a soft regular open set in $Y$ then $f-1$ pu $(G, K)$ is soft $\beta$-open over X. Since (A, E) is soft open over $\mathrm{X}$, By lemma $2(\mathrm{~A}, \mathrm{E}) \cap f_{p u}^{-1}(\mathrm{G}, \mathrm{K})=[\mathrm{fpu} /(\mathrm{A}, \mathrm{E})]-1(\mathrm{G}, \mathrm{K})$ is soft $\beta$-open in $(\mathrm{A}, \mathrm{E})$. Therefore, fpu/ $(\mathrm{A}, \mathrm{E})$ is soft almost $\beta$-continuous.

\section{Soft Almost $\beta$-Open Mappings}

Definition 1: A soft mapping fpu: $(\mathrm{X}, \tau, \mathrm{E}) \rightarrow(\mathrm{Y}, \vartheta, \mathrm{K})$ is said to be soft almost $\beta$-open if for each soft regular open set $(F, E)$ over $X, f p u(F, E)$ is soft $\beta$-open over $Y$.

Remark: Every soft $\beta$-open mapping is soft almost $\beta$-open but the converse may not be true.

Example 1: Let $X=\{x 1, x 2\}, E=\{e 1, e 2\}$ and $Y=\{y 1, y 2\}, K=\{k 1, k 2\}$. The soft sets $(F, E),(G, K)$ are defined as follows: $\mathrm{F}(\mathrm{e} 1)=\{\mathrm{x} 1\}, \mathrm{F}(\mathrm{e} 2)=\{\mathrm{x} 2\}, \mathrm{G}(\mathrm{k} 1)=\{\mathrm{y} 2\}, \mathrm{G}(\mathrm{k} 2)=\{\mathrm{y} 1\}$. Let $\tau=\{\varphi,(\mathrm{F}, \mathrm{E}), \mathrm{X}\}$ and $v=\{\varphi,(\mathrm{G}, \mathrm{K}), \mathrm{Y}\}$ are topologies on $\mathrm{X}$ and $\mathrm{Y}$ respectively. Then soft mapping fpu: $(X, \tau, \mathrm{E}) \rightarrow(\mathrm{Y}, \mathrm{v}, \mathrm{K})$ defined by $\mathrm{u}(\mathrm{x} 1)=\mathrm{y} 1, \mathrm{u}(\mathrm{x} 2)=\mathrm{y} 2$ and $\mathrm{p}(\mathrm{e} 1)=\mathrm{k} 1, \mathrm{p}(\mathrm{e} 2)=\mathrm{k} 2$ is soft almost $\beta$-open mapping but not soft $\beta$-open.

Remark: Every soft almost semi-open mapping is soft almost $\beta$-open but the converse may not be true.

Example 2: Let $\mathrm{X}=\{\mathrm{x} 1, \mathrm{x} 2\}, \mathrm{E}=\{\mathrm{e} 1, \mathrm{e} 2\}$ and $\mathrm{Y}=\{\mathrm{y} 1, \mathrm{y} 2\}, \mathrm{K}=\{\mathrm{k} 1, \mathrm{k} 2\}$. The soft sets $(\mathrm{F} 1, \mathrm{E})$ and $(\mathrm{F} 2, \mathrm{E})$ are defined as follows: $\mathrm{F} 1(\mathrm{e} 1)=\{\mathrm{x} 1\}, \mathrm{F} 1(\mathrm{e} 2)=\{\mathrm{x} 2\}, \mathrm{F} 2(\mathrm{e} 1)=\{\mathrm{x} 2\}, \mathrm{F} 2(\mathrm{e} 2)=\{\mathrm{x} 1\}$, Let $\tau=\{\varphi,(\mathrm{F} 1, \mathrm{E}),(\mathrm{F} 2, \mathrm{E}), \mathrm{X}\}$ and $v$ $=\{\varphi, \mathrm{Y}\}$ are topologies on $\mathrm{X}$ and $\mathrm{Y}$ respectively. Then soft mapping fpu: $(\mathrm{X}, \tau, \mathrm{E}) \rightarrow(\mathrm{Y}, \mathrm{v}, \mathrm{K})$ defined by $\mathrm{u}(\mathrm{x} 1)=\mathrm{y} 1$, $\mathrm{u}(\mathrm{x} 2)=\mathrm{y} 2$ and $\mathrm{p}(\mathrm{e} 1)=\mathrm{k} 1, \mathrm{p}(\mathrm{e} 2)=\mathrm{k} 2$ is soft almost $\beta$-open mapping but not soft almost semi-open.

Remark: Every soft almost pre-open mapping is soft almost $\beta$-open but the converse may not be true.

Example 3: Let $X=\{x 1, x 2\}, E=\{e 1, e 2\}$ and $Y=\{y 1, y 2\}, K=\{k 1, k 2\}$. The soft sets (F1, E), (F2, E), (G1, K), (G2, $\mathrm{K})$ and $(\mathrm{G} 3, \mathrm{~K})$ are defined as follows: $\mathrm{F} 1(\mathrm{e} 1)=\{\mathrm{x} 1\}, \mathrm{F} 1(\mathrm{e} 2)=\{\mathrm{x} 2\}, \mathrm{F} 2(\mathrm{e} 1)=\{\mathrm{x} 2\}, \mathrm{F} 2(\mathrm{e} 2)=\{\mathrm{x} 1\}, \mathrm{G} 1(\mathrm{k} 1)=\varphi, \mathrm{G} 1(\mathrm{k} 2)$ $=\{\mathrm{y} 1\}, \mathrm{G} 2(\mathrm{k} 1)=\{\mathrm{y} 1\}, \mathrm{G} 2(\mathrm{k} 2)=\varphi, \mathrm{G} 3(\mathrm{k} 1)=\{\mathrm{y} 1\}, \mathrm{G} 3(\mathrm{k} 2)=\{\mathrm{y} 1\}$. Let $\tau=\{\varphi,(\mathrm{F} 1, \mathrm{E}),(\mathrm{F} 2, \mathrm{E}), \mathrm{X}\}$ and $v=\{\varphi,(\mathrm{G} 1, \mathrm{~K})$, $(\mathrm{G} 2, \mathrm{~K}),(\mathrm{G} 3, \mathrm{~K}), \mathrm{Y}\}$ are topologies on $\mathrm{X}$ and $\mathrm{Y}$ respectively. Then soft mapping fpu: $(\mathrm{X}, \tau, \mathrm{E}) \rightarrow(\mathrm{Y}, v, \mathrm{~K})$ defined by $\mathrm{u}(\mathrm{x} 1)=\mathrm{y} 1, \mathrm{u}(\mathrm{x} 2)=\mathrm{y} 2$ and $\mathrm{p}(\mathrm{e} 1)=\mathrm{k} 1, \mathrm{p}(\mathrm{e} 2)=\mathrm{k} 2$ is soft almost $\beta$-open mapping but not soft almost pre-open.

Remark: Every soft almost $\alpha$-open mapping is soft almost semi-open but the converse may not be true.

Example 4: Let $X=\{\mathrm{x} 1, \mathrm{x} 2\}, \mathrm{E}=\{\mathrm{e} 1, \mathrm{e} 2\}$ and $\mathrm{Y}=\{\mathrm{y} 1, \mathrm{y} 2\}, \mathrm{K}=\{\mathrm{k} 1, \mathrm{k} 2\}$. The soft sets (F1, E), (F2, E), (G1, K), (G2, $\mathrm{K})$ and $(\mathrm{G} 3, \mathrm{~K})$ are defined as follows : $\mathrm{F} 1(\mathrm{e} 1)=\{\mathrm{x} 1\}, \mathrm{F} 1(\mathrm{e} 2)=\{\mathrm{x} 2\}, \mathrm{F} 2(\mathrm{e} 1)=\{\mathrm{x} 2\}, \mathrm{F} 2(\mathrm{e} 2)=\{\mathrm{x} 1\}, \mathrm{G} 1(\mathrm{k} 1)=\varphi$, $\mathrm{G} 1(\mathrm{k} 2)=\{\mathrm{y} 1\}, \mathrm{G} 2(\mathrm{k} 1)=\{\mathrm{y} 1\}, \mathrm{G} 2(\mathrm{k} 2)=\varphi, \mathrm{G} 3(\mathrm{k} 1)=\{\mathrm{y} 1\}, \mathrm{G} 3(\mathrm{k} 2)=\{\mathrm{y} 1\}$. Let $\tau=\{\varphi,(\mathrm{F} 1, \mathrm{E}),(\mathrm{F} 2, \mathrm{E}), \mathrm{X}\}$ and $v=\{\varphi$, $(\mathrm{G} 1, \mathrm{~K}),(\mathrm{G} 2, \mathrm{~K}),(\mathrm{G} 3, \mathrm{~K}), \mathrm{Y}\}$ are topologies on $\mathrm{X}$ and $\mathrm{Y}$ respectively. Then soft mapping fpu: $(\mathrm{X}, \tau, \mathrm{E}) \rightarrow(\mathrm{Y}, v, \mathrm{~K})$ defined by $u(x 1)=y 1, u(x 2)=y 2$ and $p(e 1)=k 1, p(e 2)=k 2$ is soft almost semi-open mapping but not soft almost $\alpha$ open.

Remark: The concepts of soft almost semi-open and soft almost pre-open mappings are independent.

Example 5: Let $X=\{x 1, x 2\}, E=\{e 1, e 2\}$ and $Y=\{y 1, y 2\}, K=\{k 1, k 2\}$. The soft sets (F1, E) and (F2, E) are defined as follows: $\mathrm{F} 1(\mathrm{e} 1)=\{\mathrm{x} 1\}, \mathrm{F} 1(\mathrm{e} 2)=\{\mathrm{x} 2\}, \mathrm{F} 2(\mathrm{e} 1)=\{\mathrm{x} 2\}, \mathrm{F} 2(\mathrm{e} 2)=\{\mathrm{x} 1\}$. Let $\tau=\{\varphi,(\mathrm{F} 1, \mathrm{E}),(\mathrm{F} 2, \mathrm{E}), \mathrm{X}\}$ and $v=$ $\{\varphi, \mathrm{Y}\}$ are topologies on $\mathrm{X}$ and $\mathrm{Y}$ respectively. Then soft mapping fpu: $(\mathrm{X}, \tau, \mathrm{E}) \rightarrow(\mathrm{Y}, v, \mathrm{~K})$ defined by $\mathrm{u}(\mathrm{x} 1)=\mathrm{y} 1$, $\mathrm{u}(\mathrm{x} 2)=\mathrm{y} 2$ and $\mathrm{p}(\mathrm{e} 1)=\mathrm{k} 1, \mathrm{p}(\mathrm{e} 2)=\mathrm{k} 2$ is soft almost pre-open but not soft almost semi-open. 
Example 6: Let $X=\{x 1, x 2\}, E=\{e 1, e 2\}$ and $Y=\{y 1, y 2\}, K=\{k 1, k 2\}$. The soft sets $(F 1, E),(F 2, E),(G 1, K),(G 2$, $\mathrm{K})$ and $(\mathrm{G} 3, \mathrm{~K})$ are defined as follows: $\mathrm{F} 1(\mathrm{e} 1)=\{\mathrm{x} 1\}, \mathrm{F} 1(\mathrm{e} 2)=\{\mathrm{x} 2\}, \mathrm{F} 2(\mathrm{e} 1)=\{\mathrm{x} 2\}, \mathrm{F} 2(\mathrm{e} 2)=\{\mathrm{x} 1\}, \mathrm{G} 1(\mathrm{k} 1)=\varphi$, $\mathrm{G} 1(\mathrm{k} 2)=\{\mathrm{y} 1\}, \mathrm{G} 2(\mathrm{k} 1)=\{\mathrm{y} 1\}, \mathrm{G} 2(\mathrm{k} 2)=\varphi, \mathrm{G} 3(\mathrm{k} 1)=\{\mathrm{y} 1\}, \mathrm{G} 3(\mathrm{k} 2)=\{\mathrm{y} 1\}$. Let $\tau=\{\varphi,(\mathrm{F} 1, \mathrm{E}),(\mathrm{F} 2, \mathrm{E}), \mathrm{X}\}$ and $v=\{\varphi$, $(\mathrm{G} 1, \mathrm{~K}),(\mathrm{G} 2, \mathrm{~K}),(\mathrm{G} 3, \mathrm{~K}), \mathrm{Y}\}$ are topologies on $\mathrm{X}$ and $\mathrm{Y}$ respectively. Then soft mapping fpu: $(\mathrm{X}, \tau, \mathrm{E}) \rightarrow(\mathrm{Y}, v, \mathrm{~K})$ defined by $u(x 1)=y 1, u(x 2)=y 2$ and $p(e 1)=k 1, p(e 2)=k 2$ is soft almost semi-open mapping but not soft almost preopen.

Thus we reach the following diagram of implications

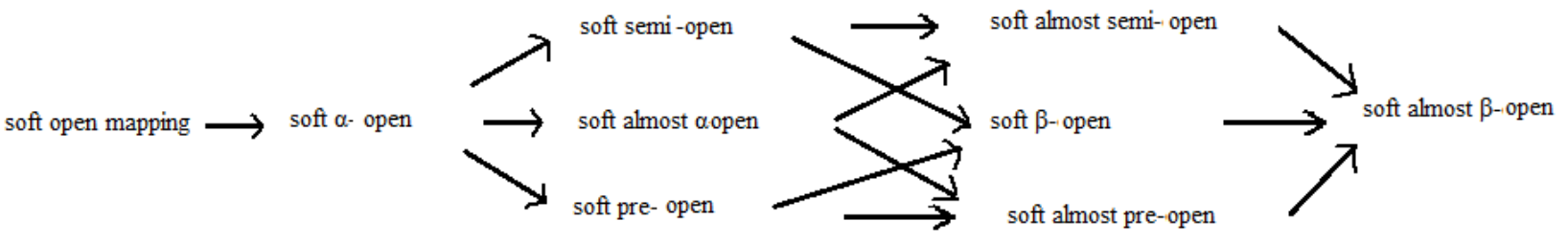

Figure 2: Soft open mapping

\section{Properties of Soft Almost $\beta$-Open Mappings}

Theorem 1: Let $\mathrm{f}_{\mathrm{plu} 1}:(\mathrm{X}, \tau, \mathrm{E}) \rightarrow(\mathrm{Y}, \vartheta, \mathrm{K})$ and $\mathrm{g}_{\mathrm{p} 2 \mathrm{u} 2}:(\mathrm{Y}, \vartheta, \mathrm{K}) \rightarrow(\mathrm{Z}, \eta, \mathrm{T})$ be two soft mappings, If $\mathrm{f}_{\mathrm{p} 1 \mathrm{u} 1}$ is soft almost open and $g_{\mathrm{p} 2 \mathrm{u} 2}$ is soft $\beta$-open. Then the soft mapping $\mathrm{g}_{\mathrm{p} 2 \mathrm{u} 2} 0 \mathrm{f}_{\mathrm{p} 1 \mathrm{u} 1}:(\mathrm{X}, \tau, \mathrm{E}) \rightarrow(\mathrm{Z}, \eta, \mathrm{T})$ is soft almost $\beta$-open.

Proof: Let $(F, E)$ be soft regular open over X. Then $f_{p l u 1}(F, E)$ is soft open over $Y$ because $f_{p l u 1}$ is soft almost open. Therefore, $g_{p 2 u 2}\left(f_{p 1 u 1}\right)(F, E)$ is soft $\beta$-open over Z. Because $g_{p 2 u 2}$ is soft $\beta$-open. Since $\left(g_{p 2 u 2} 0 f_{p 1 u 1}\right)(F, E)=\left(g_{p 2 u 2}\left(f_{p 1 u 1}\right.\right.$ $(\mathrm{F}, \mathrm{E}))$, it follows that the soft mapping $\left(\mathrm{g}_{\mathrm{p} 2 \mathrm{u} 2}\right.$ of $\left.\mathrm{p}_{\mathrm{p} 1 \mathrm{u} 1}\right)$ is soft almost $\beta$-open.

Definition 1: A soft mapping fpu: $(\mathrm{X}, \tau, \mathrm{E}) \rightarrow(\mathrm{Y}, \vartheta, \mathrm{K})$ is said to be soft $\beta$-irresolute if the inverse image of soft $\beta$-open set of $\mathrm{Y}$ is soft $\beta$-open set over $\mathrm{X}$.

Theorem 2: Let $\mathrm{f}_{\mathrm{p} 1 \mathrm{u} 1}:(\mathrm{X}, \tau, \mathrm{E}) \rightarrow(\mathrm{Y}, \vartheta, \mathrm{K})$ and $\mathrm{g}_{\mathrm{p} 2 \mathrm{u} 2}:(\mathrm{Y}, \vartheta, \mathrm{K}) \rightarrow(\mathrm{Z}, \eta, \mathrm{T})$ be two soft mappings, such that $\mathrm{g}_{\mathrm{p} 2 \mathrm{u} 2} 0 \mathrm{f}_{\mathrm{p} 1 \mathrm{u} 1}$ : $(X, \tau, E) \rightarrow(Z, \eta, T)$ is soft almost $\beta$-open and $g_{p 2 u 2}$ is soft $\beta$-irresolute and injective then $f_{p 1 u 1}$ is soft almost $\beta$-open.

Proof: Suppose (F, E) is soft regular open set over X. Then $g_{\mathrm{p} 2 \mathrm{u} 2} 0 \mathrm{fp}_{1} \mathrm{u}_{1}(\mathrm{~F}, \mathrm{E})$ is soft $\beta$-open over $Z$ because $\mathrm{g}_{\mathrm{p} 2 \mathrm{u} 2} 0 \mathrm{fp}_{1} \mathrm{u}_{1}$ is soft almost $\beta$-open. Since $g_{\mathrm{p} 2 \mathrm{u} 2}$ is injective, we have $\left(\mathrm{g}-1 \mathrm{p} 2 \mathrm{u} 2\left(\mathrm{~g}_{\mathrm{p} 2 \mathrm{u} 2} 0 \mathrm{fp}_{1} \mathrm{u}_{1}\right)(\mathrm{F}, \mathrm{E})\right)=\mathrm{fp}_{1} \mathrm{u}_{1}(\mathrm{~F}, \mathrm{E})$. Therefore $\mathrm{fp}_{1} \mathrm{u}_{1}(\mathrm{~F}$, $E)$ is soft $\beta$-open in $Y$ because $g_{p 2 u 2}$ is soft $\beta$-irresolute. This implies $\mathrm{fp}_{1} \mathrm{u}_{1}$ is soft almost $\beta$-open.

Theorem 2: Let soft mapping fpu: $(\mathrm{X}, \tau, \mathrm{E}) \rightarrow(\mathrm{Y}, \vartheta, \mathrm{K})$ be soft almost $\beta$-open mapping. If $(\mathrm{G}, \mathrm{K})$ is soft set of $\mathrm{Y}$ and $(\mathrm{F}$, $\mathrm{E})$ is soft regular closed set of $\mathrm{X}$ containing $f_{p u}^{-1}(\mathrm{G}, \mathrm{K})$ then there is a soft $\beta$-closed set $(\mathrm{A}, \mathrm{K})$ of $\mathrm{Y}$ containing $(\mathrm{G}, \mathrm{K})$ such that $f_{p u}^{-1}(\mathrm{~A}, \mathrm{~K}) \subset(\mathrm{F}, \mathrm{E})$.

Proof: Let $(A, K)=(f p u(F, E) C) C$. Since $f_{p u}^{-1}(G, K) \subset(F, E)$, we have fpu(F, E) C $\subset(G, K)$. Since fpu is soft almost $\beta$-open then $(\mathrm{A}, \mathrm{K})$ is soft $\beta$-closed set over $\mathrm{Y}$ and $f_{p u}^{-1}(\mathrm{~A}, \mathrm{~K})=f_{p u}^{-1}(\mathrm{fpu}(\mathrm{F}, \mathrm{E}) \mathrm{C}) \mathrm{C} \subset((\mathrm{F}, \mathrm{E}) \mathrm{C}) \mathrm{C}=(\mathrm{F}, \mathrm{E})$. Thus, $f_{p u}^{-1}$ $(\mathrm{A}, \mathrm{K}) \subset(\mathrm{F}, \mathrm{E})$.

\section{REFERENCES}

1. Error! Reference source not found.Error! Reference source not found.Error! Reference source not found.Error! Reference source not found.Error! Reference source not found.Error! Reference source not found.Error! Reference source not found.Error! Reference source not found.Akdag, M. \& Ozkan, A. (2014A). On soft preopen sets and soft pre separation axioms. GU J Sci., 27(4), 1077-1083.

2. Akdag, M. \& Ozkan, A. (2014B). On soft $\alpha$-open sets and soft $\alpha$-continuous functions. Abstract and Applied Analysis 2014, Article ID 891341, pp. 7. https://doi.org/10.1155/2014/891341

3. Akdag, M. \& Ozkan, A. (2014C). On Soft $\beta$-Open Sets and Soft $\beta$-continuous functions. The Scientific World Journal, Article ID 843456, pp. 6. https://doi.org/10.1155/2014/843456

4. Ali, I. M., Feng, F., Liu, X., Min, W. K. \& Shabir, M. (2009). On some new operations in soft set theory. Comput. Math. Appl. 57, 1547-1553. https://doi.org/10.1016/j.camwa.2008.11.009

5. Arockiarani, I. \& Lancy, A. A. (2013). Generalized soft g $\beta$ - closed sets and soft gs $\beta$-closed sets in soft topological spaces. International Journal of Mathematical Archive, 4(2), 1-7.

6. Chen, B. (2013). Soft semi-open sets and related properties in soft topological spaces. Appl. Math. Inf. Sci., 7(1), 287-294. https://doi.org/10.12785/amis/070136

7. Hussain, S. \& Ahmad, B. (2011). Some properties of soft topological spaces. Comput. Math. Appl., 62, 40584067. https://doi.org/10.1016/j.camwa.2011.09.051 
8. Kharral, A. \& Ahmad, B. (2011). Mappings on soft classes. New Math. Nat. Comput., 7(3), 471-481. https://doi.org/10.1142/S1793005711002025

9. Mahanta, J. \& Das, P. K. (2014). On soft topological space via semi open and semi closed soft sets. Kyungpook Math. J. 54, 221-236. https://doi.org/10.5666/KMJ.2014.54.2.221

10. Maji, P. K., Biswas, R. \& Roy, R. (2003). Soft set theory. Comput. Math. Appl., 45, 555-562. https://doi.org/10.1016/S0898-1221(03)00016-6

11. Majumdar, P. \& Samanta, S. K. (2008). Similarity measure of soft sets. New Math. Nat. Comput. 4(1), 1-12. https://doi.org/10.1142/S1793005708000908

12. Min, W. K. (2011). A note on soft topological spaces. Comput. Math. Appl., 62, 3524-3528. https://doi.org/10.1016/j.camwa.2011.08.068

13. Molodtsov, D. (1999). Soft set theory first results. Comput. Math. Appl., 37, 19-31. https://doi.org/10.1016/S0898-1221(99)00056-5

14. Saziye, Y. (2014). Soft Regular Generalized Closed Sets in Soft Topological Spaces. Int. Journal of Math. Analysis, 8(8), 355-367. https://doi.org/10.12988/ijma.2014.4125

15. Shabir, M. \& Naz, M. (2011). On soft topological spaces. Comput. Math. Appl., 61, $1786-1799$. https://doi.org/10.1016/j.camwa.2011.02.006

16. Thakur S.S. \& Rajput, A.S. (2017B). Soft Almost Semi-Continuous Mappings. Malaya J. Mat., 5(2), 395-400.

17. Thakur S.S. \& Rajput, A.S. (2018). Soft Almost Pre-Continuous Mappings. The Journal of Fuzzy Mathematics, 26(2), 439-449. https://doi.org/10.20454/jast.2018.1415

18. Thakur, S.S. \& Rajput, A.S. (2017A). Soft Almost Continuous Mappings. Inter. Jr. of Adv. in Math., 1, $22-29$.

19. Thakur, S.S. \& Rajput, A.S. (2019). Extremally Disconnectedness in Soft Topological Spaces. The Journal of Fuzzy Mathematics, 27(2) 1-25.

20. Yumak, Y. \& Kayamakci, A.K. (2013). Soft beta-open sets and their application. arXiv:1312.6964.

21. Zorlutana, I., Akdag, N. \& Min, W.K. (2012). Remarks on soft topological spaces. Ann. Fuzzy Math. Inf., 3(2), 171-185.

22. Zorlutuna, I. \& Hatice, C. (2015). On Continuity of Soft Mappings Appl. Math. Inf. Sci. 9(1), 403-409. https://doi.org/10.12785/amis/090147 